\section{Salmonella Abscess in a Failed Transplanted Kidney}

\author{
Heath Catoe ${ }^{1}$, Jeremy Ramdial ${ }^{2}$, Adriana Dejman ${ }^{3 *}$ and \\ Warren Kupin ${ }^{4}$
}

${ }^{1}$ Department of Internal Medicine, Jackson Memorial Hospital, Miami, FL, USA

${ }^{2}$ Department of Hematology and Oncology, Jackson Memorial Hospital, Miami, FL, USA

${ }^{3}$ Department of Nephrology, Jackson Memorial Hospital, Miami, FL, USA

${ }^{4}$ Department of Nephrology, The University of Miami Health System, Miami, FL, USA

\section{Abstract}

This case report details the rare occurrence of Salmonella abscess in a failed kidney transplant. A 53-year-old Jamaican American woman with diabetes type 2 back on dialysis after failure of a kidney transplant presented with a 2 month history of decreased appetite and fatigue subsequently developing malaise and fevers. Evaluation with MRI showed an abscess that engulfed the previous kidney transplant location. After percutaneous drainage, culture of abscess grew Salmonella enteric subspecies sero type Group O: 47,48 or 50 . The patient was sent home on IV antibiotics with subsequent follow-up and drain removal. Although Salmonella abscesses in failed kidney transplant are rare, appropriate treatment can be conservative, similar to other renal abscesses.

Keywords: Salmonella abscess; Kidney transplant; Diabetes failed
\end{abstract} kidney transplant; Kidney abscess drainage

\section{Background}

An abscess in the kidney is due to salmonellae are rare in the literature and rarer in kidney-transplant patients. The etiology of such abscesses can be difficult to decipher. Salmonella enteric subspecies I serotypes can cause disease in a range of hosts depending on their adaptations. Human host restricted serotypes of typhi, paratyphi A and sendai tend to cause more systemic infections as they are thought to be more adapted to their host. Unrestricted host Salmonella species usually include typhirium and enteritidis and mostly cause self-limited gastroenteritis symptoms but in a range of higher vertebrates [1]. The CDC estimates there are 1.2 million Salmonella infections per year with surveillance data indicating Salmonella serovar enteritidis being the most common serotype followed by Salmonella serovar

*Corresponding author: Adriana Dejman, Department of Nephrology, Jackson Memorial Hospital, Miami, FL, USA, Tel: +1 3052438491; E-mail: adriana.dejman@jhsmiami.org

Citation: Catoe H, Ramdial J, Dejman A, Kupin W, (2017) Salmonella Abscess in a Failed Transplanted Kidney. J Infect Non Infect Dis 3: 019.

Received: October 24, 2016; Accepted: January 12, 2017; Published: January 26, 2017 typhimurium and Salmonella serovar newport. Chicken eggs are known source of these salmonellae outbreaks [2,3]. The patient's history presents a compelling possibility of a previous systemic infection of Salmonella prior to presentation that could have seeded her kidney transplant leading its destruction via abscess. Although full sero typing was not completed, $\mathrm{O}$ antigen reactivity did not indicate it was from one of the common subspecies found on surveillance data. Successful treatment included systemic antibiotics with percutaneous drainage. This case report details the rare possibility of a systemic Salmonella infection seeding and destroying a failed kidney graft with subsequent management.

\section{Case Presentation}

53-year-old Jamaican woman with history of hypertension, diabetes mellitus type 2 and end-stage renal disease presented to an outside hospital in December 2013. The patient was admitted with altered mental status, fevers, abdominal pain, fatigue and anorexia ongoing for two months, but without diarrhea. Her graft history dates to 2007 after receiving a deceased donor kidney transplant but failed after graft rejection and resumed hemodialysis in February 2013. Her remaining immunosuppression at time of presentation was prednisone. After resuscitation, she recovered mental status, but abdominal pain persisted which prompted imaging with an MRI showing a fluid collection and air pockets in the right lower quadrant of the abdomen, abutting the right psoas muscle. The patient was started on vancomycin at that time due to concern for Staphylococcus aureus. CT guided abscess drainage on day 5 of hospitalization retrieved $20 \mathrm{~mL}$ of purulent material that was sent for gram stain and culturing. Gram stain showed 5-10 WBCs (1+) and rare 1-2 gram positive cocci per slide; however, despite no gram negative rods in the smear, few colonies of Salmonella grew from automated aerobic cultures on proprietary media. The patient was changed to ciprofloxacin $200 \mathrm{mg}$ BID IV on approximately day $8^{\text {th }}$ based on Salmonella sensitivities. She was a febrile for the remainder of her hospital course. Final sero typing on day 16 indicated Salmonella enterica subspecies sero type Group O: 47, 48, or 50. Unfortunately due to time and outside laboratory circumstances, $\mathrm{H}$ antigen was not performed and it is unclear if the sample was lost in submission to the California health department. Although this polyvalent sera test indicated $\mathrm{O}$ antigens from all subgenera, it contained only the several rare forms of Salmonella enterica subspecies enterica associated with human infections. Blood cultures were negative throughout her hospital course. Acid fast bacillus and fungal cultures were also negative. She was then transported to our facility on day 17. Upon transfer, investigation revealed a total WBC count of $10,900 /$ mcL with an absolute Neutrophil count of 7,300/mcL. Urinalysis was not done since the patient was anuric. CT scan with contrast confirmed abscess in the renal transplant displaying no apparent kidney parenchyma and a $5.9 \times 5.5 \times 10.0 \mathrm{~cm}$ collection (Figure 1).

No other collections or sites of infection were identified. Blood cultures and repeated catheter drainage cultures were negative with patient remaining afebrile. We continued her hemodialysis and adjusted ciprofloxacin dose to 400mg IV QD for dosing convenience. The 


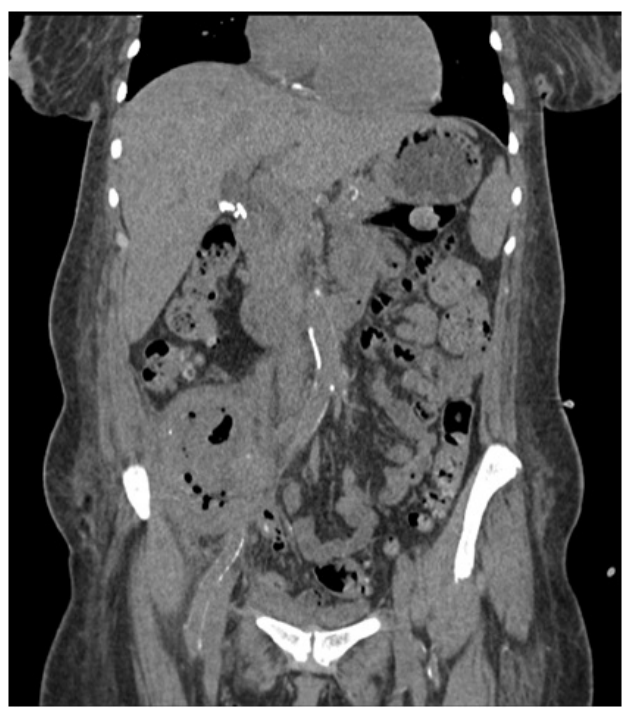

Figure 1: CT scan coronal view of abdomen showing transplanted kidney with air cavities and ablation of normal parenchyma.

patient retained a cavity approximately $10 \mathrm{cc}$ in size that was drained and lavaged with debridement (Figure 2A).

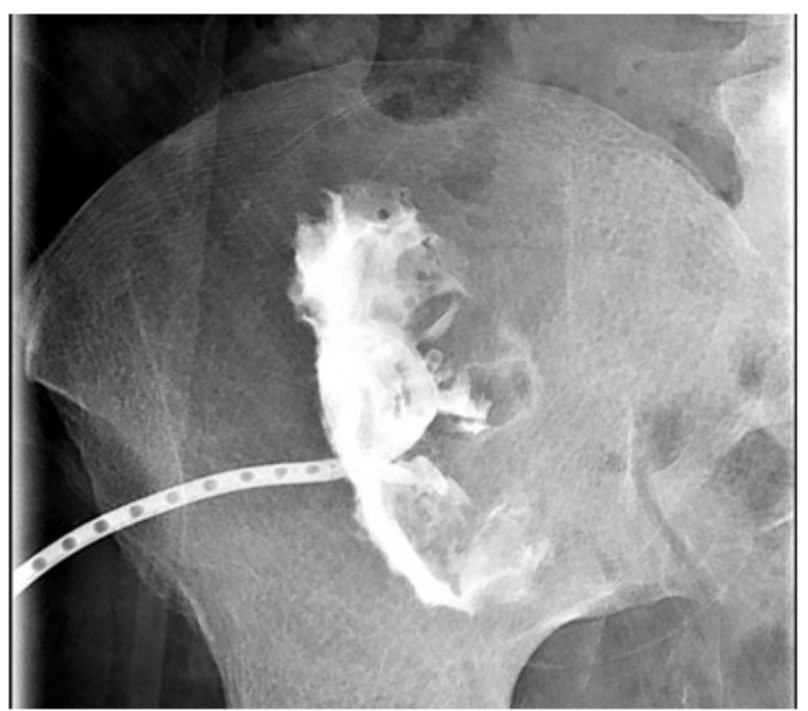

Figure 2A: Abscessogram demonstrating 10cc cavity 2 weeks after discharge.

Surgical resection was discussed but it would be a high risk vascular surgery as opposed to continued drainage with antibiotics. Patient was switched to IV ceftriaxone 2g QDon day 23 for 6 weeks via peripherally inserted central catheterline for easier compliance and sent home on day $25^{\text {th }}$ after drain tube exchanged. Abscessogram approximately 3 months after admission showed an $8 c c$ cavity and drain was removed for a trial period (Figure 2B). The patient has no recorded recurrence of infection for several months.

\section{Discussion}

Graft abscesses are uncommon and further Salmonella infections are scarce. It is proposed that she had a previous undetected Salmonella infection that became systemic and seeded the graft, however, the mechanism of infection remains uncertain. It may be speculated that infection arose from her increased egg intake as a source, but it remains dubious. Although the serotype did not indicate the more

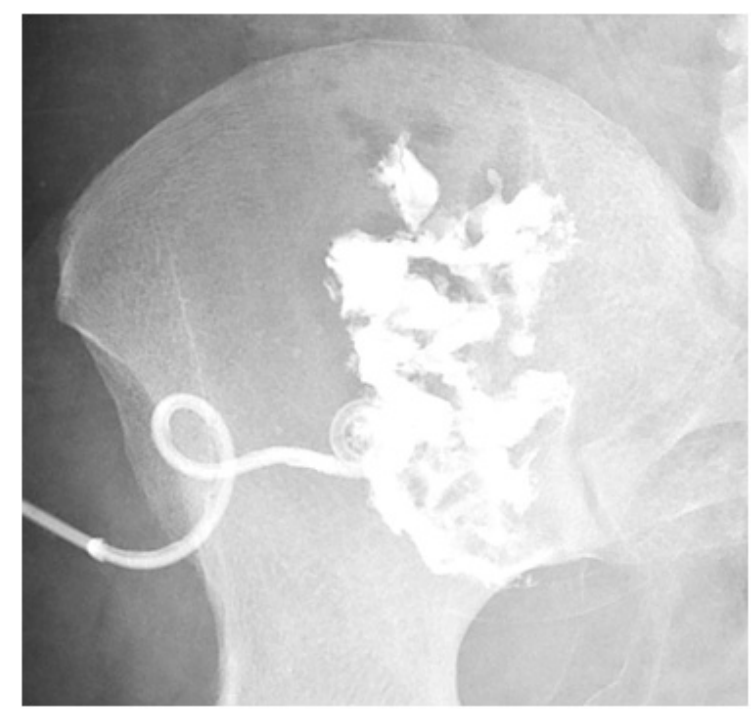

Figure 2B: An 8cc cavity remaining 3 months after discharge.

common subspecies of Salmonella, her clinical picture indicates a more host adapted Salmonella infection. Host adapted infections like typhi usually proceeds with oral ingestion resulting in invasion of mucosal without significant inflammation to cause enteritis. From there, one could imagine Salmonella surviving in the polymorphonuclear leukocyte to disseminate in the lymphatics [1]. Although retained grafts such as in our patient may have a protective effect from systemic infections, it may have been the localizing factor for this infection. Retained grafts have been shown to reduce sepsis related mortality and systemic infections thought to be due in part to the systemic inflammatory state induced in the host [4-6]. But this intense inflammation site could have attracted infected macrophages, thus seeding the transplanted kidney.

An alternative etiology hypothesis is that she may have contracted the infection through hemodialysis. Bacteremia is higher in patients on hemodialysis which could have lead to seeding, but Staphylococcus aureus is the major pathogen associated with hemodialysis and this was not detected in our patient [7]. On the contrary, her risk for infection in general may have been increased by several other factors.

Immunosuppression after renal allograft rejection shows decreased risk of sensitization at the expense of an increased risk of infections [8]. However our patient had been off antimetabolites and calcineurin inhibitors for almost one year. Although she may have had some protective factors in the retained kidney transplant, our patient was at an increased risk for bacterial infections and renal abscesses in particular as she is diabetic which leads to increased urinary tract infections and impairment of immune system functions [9-12].

Salmonella abscesses are rare and reported in only a few case reports [13-22]. The literature shows that treatment can range from conservative to invasive for renal Salmonella abscesses [23-26]. Management varied but usual treatment included percutaneous drainage with susceptible antibiotic treatment by IV or PO of antibiotics ceftriaxone, ciprofloxacin and evenofloxacin. In one case, percutaneous drainage failed with treatment proceeding to nephrectomy with 2 weeks postop antibiotics of ciprofloxacin. However, to our knowledge this is the first case report of a renal abscess due to Salmonella in a failed kidney graft. Due to ongoing infection with a risk of seeding, surgical removal was not done. Our patient was treated with percutaneous drainage and antibiotics with a good response. 
Appropriate management of renal abscesses in general is more clearly defined than specifically Salmonella renal abscess. Before the widespread use of antibiotics, S. aureus was the predominant organism causing renal abscesses; however, the majority of organisms now are gram negative [27]. One current scheme for treatment of renal abscess is based on size with a $3-5 \mathrm{~cm}$ renal abscess being amenable to multiple weeks of IV antibiotics alone; however this is considered in immunocompetent patients [28-30]. Elevation of treatment would be percutaneous drainage which has a high cure rate and is usually considered in abscesses larger than $5 \mathrm{~cm}$ [10,28,31-33]. Larger than 5 $\mathrm{cm}$ is associated with increased need for surgical drainage or multiple percutaneous drainage procedures [29]. Several factors are associated with antimicrobial treatment failure including large abscess, obstructive uropathy, severe vesicoureteral reflux, diabetes, old age and urosepsis with gas forming organisms [12]. Drainage should be considered if no clinical improvement occurs in 2-3 days of appropriate antibiotics [12].

In patients, especially renal transplant recipients, renal abscess due to salmonellae is a rare occurrence. This rare case demonstrates a renal abscess in immunosuppressed patients with a kidney transplant can have rare microbiologic etiology. Further conservative management with percutaneous drainage and antimicrobials could potentially be curative. We corroborate that management of a failed renal graft is an important part of renal transplant planning and treatment.

\section{References}

1. Uzzau S, Brown DJ, Wallis T, Rubino S, Leori G, et al. (2000) Host adapted serotypes of Salmonella enterica. Epidemiol Infect 125: 229-255.

2. Centers for Disease Control and Prevention (2010) Preliminary Food Net data on the incidence of infection with pathogens transmitted commonly through food - 10 states, 2009. Morbidity and mortality weekly report 59: 418-422.

3. Hohmann EL (2001) Nontyphoidal salmonellosis. Clinical infectious diseases 32: $263-269$.

4. Lopez Gomez JM, Perez Flores I, Jofre R, Carretero D, Rodriguez Benitez et al. (2004) Presence of a failed kidney transplant in patients who are on hemodialysis is associated with chronic inflammatory state and erythropoietin resistance. J Am Soc Nephrol 15: 2494-2501.

5. Marcen R, Teruel JL (2008) Patient outcomes after kidney allograft loss. Transplantation Rev 22: 62-72.

6. Ayus JC, Achinger SG, Lee S, Sayegh MH, Alan S Go (2010) Transplant nephrectomy improves survival following a failed renal allograft. J Am Soc Nephrol 21: 374-380.

7. Lewis SS, Sexton DJ (2013) Metastatic complications of bloodstream infections in hemodialysis patients. Semin Dial 26: 47-53.

8. Woodside KJ, Schirm ZW, Noon KA, Huml AM, Padiyar A, et al. (2014) Fever, infection, and rejection after kidney transplant failure. Transplantation 97: 648-653.

9. Abbott KC, Oliver JD3rd, Hypolite I, Lepler LL, Kirk AD, et al. (2001) Hospitalizations for bacterial septicemia after renal transplantation in the united states. Am J Nephrol 21: 120-127.

10. Coelho RF, Schneider Monteiro ED, Mesquita JL, Mazzucchi E, Marmo Lucon A, et al. (2007) Renal and perinephric abscesses: analysis of 65 consecutive cases. World J Surg 31: 431-436.

11. Dharnidharka VR, Agodoa LY, Abbott KC (2007) Risk factors for hospitalization for bacterial or viral infection in renal transplant recipients--an analysis of USRDS data. Am J Transplant :7: 653-661.
12. Yen DH, Hu SC, Tsai J, Kao WF, Chern CH, et al. (1999) Renal abscess: early diagnosis and treatment. Am J Emerg Med 17: 192-197.

13. Borrero GO, Jafri SZ, Vazquez PM, Hollowell ML, Kling GA (1986) Computed tomography of acute renal abscess due to Salmonella chester. Comput Radiol 10: 41-44.

14. Davis NS, Powell KR, Rabinowitz R (1989) Salmonella renal abscess in a four-year-old child. Pediatr Infect Dis J 8: 122-123.

15. D'Cruz S, Kochhar S, Chauhan S, Gupta V (2009) Isolation of Salmonella paratyphi A from renal abscess. Indian J Pathol Microbiol 52: 117-119.

16. Dravid M, Joshi BN (1990) Renal abscess due to Salmonella typhimurium. Indian J Pediatr 57: 586-588.

17. Hashino S, Imamura M, Tanaka J, Noto S, Kobayashi S, et al. (1996) Renal Salmonella enteritidis abscess in a patient with severe aplastic anemia after allogeneic stem cell transplantation. Bone Marrow Transplant 18: 807-808.

18. Ikeda R, Suzuki K, Tanaka T, Tsugawa R (1985) Four cases of Salmonella urinary tract infection. Hinyokika Kiyo 31: 1199-1202.

19. Olson ES, Asmussen T, Vicca AF, Osborn DE (1999) A case report of renal abscess caused by Salmonella virchow phage type 1 associated with a papillary renal cell carcinoma. J Infect 38: 56-57.

20. Rao RS, Sharma S (1984) Uncommon manifestations of salmonellosis. Indian J Med Sci 38: 250-253.

21. Rus RR, Kersnik Levart T (2010) Acute pyelonephritis with renal abscesses and acute renal failure after salmonella infection. Acta paediatrica 99: 470473.

22. Zaludko J, Zaludkova L, Jakubciak J, Demkova A, Hlaucova Z (1970) [Salmonella choleraesuis as the cause of paranephritic abscess]. BratisI Lek Listy 53: 349-352.

23. Kaneti J, Hertzanu Y (. 1987) Renal abscess owing to salmonella septicemia: percutaneous drainage. J Urol 138: 395-396.

24. Oliver RM, Waller DG, Rubin CM, Booth LV (1990) Splenic and renal abscesses following Salmonella virchow septicaemia--conservative management with ciprofloxacin. Br J Clin Pract 44: 670-671.

25. Rai RS, Karan SC, Kayastha SM (2007) Renal and perinephric abscesses revisited. Med J Armed Forces India 63: 223-225.

26. Wippermann CF, Schofer O, Beetz R, Schumacher R, Schweden F, et al. (1991) Renal abscess in childhood: diagnostic and therapeutic progress. $\mathrm{Pe}-$ diatr Infect Dis J 10: 446-450.

27. Saiki J, Vaziri ND, Barton C (1982) Perinephric and intranephric abscesses: a review of the literature. West J Med 136: 95-102.

28. Lee SH, Jung HJ, Mah SY, Chung BH (2010) Renal abscesses measuring $5 \mathrm{~cm}$ or less: outcome of medical treatment without therapeutic drainage. Yonsei Med J 51: 569-573.

29. Siegel JF, Smith A, Moldwin R (1996) Minimally invasive treatment of renal abscess. J Urol 155: 52-55.

30. Lee BE, Seol HY, Kim TK, Seong EY, Song SH, et al. (2008) Recent clinical overview of renal and perirenal abscesses in 56 consecutive cases. Korean J Intern Med 23: 140-148.

31. Deyoe LA, Cronan JJ, Lambiase RE, Dorfman GS (1990) Percutaneous drainage of renal and perirenal abscesses: results in 30 patients. AJR Am J Roentgenol 155: 81-83.

32. Kropfl D, Behrendt H, Ringert RH, Eickenberg HU (1980) [Surgical procedure for salmonellosis of the urogenital tract (author's transl)]. Infection 8: 303-304.

33. Lang EK (1990) Renal, perirenal, and pararenal abscesses: percutaneous drainage. Radiology 174: 109-113. 